\title{
Les apports solides de l'Arve dans le Rhône genevois
}

\author{
M. Cohen, Ph. Briod \\ Genève (Suisse)
}

\section{Les chasses des biefs amont des usines de Chèvres 1896-1942 et Verbois 1942-1988}

\subsection{L'Arve, rivière alluvionnante}

Cette rivière dont la vallée est en cours de creusement, transporte des quantités de matériaux estimées par différentes méthodes mais généralement reconnues comme importantes. Des évaluations ont été faites en 1915 ; elles indiquent pour les matériaux en suspension 1,6 million de $\mathrm{m}^{3}$; les graviers charriés se montaient à l'époque à $160000 \mathrm{~m}^{3}$. Ces chiffres sont des valeurs moyennes annuelles, nous n'avons pas d'indication sur les méthodes de mesure.

Au stade de la conception et plus tard de l'exploitation des ouvrages de Chèvres et Verbois, une préoccupation très large a été accordée au phénomène redouté du comblement du bief amont par les alluvions de l'Arve. On admettait à cette époque qu'il était capital d'élucider complètement de quelle manière on pourrait évacuer les dépôts de limon.

La création de la retenue artificielle de Chèvres (plus tard celle de Verbois) provoque un ralentissement des eaux et opère un bouleversement important du régime de transport des alluvions.

Le remous de la retenue situé à la Jonction (confluent de l'Arve dans le Rhône) entraîne la formation d'un seuil constitué des plus grosses pièces de gravier (15 à $30 \mathrm{~kg}$ ). Ce seuil marque un relèvement du niveau de l'Arve à la Jonction avec des répercussions sur la nappe phréatique qui est très haute, ce qui a pour conséquence de noyer les structures inférieures des immeubles de ce quartier. Ainsi, le maintien d'un niveau constant à la Jonction reste le soucis permanent en phase d'exploitation des débits du Rhône ; c'est aussi l'effet principal escompté par les chasses.

\subsection{L'usine de Chèvres; les chasses (1)}

A l'origine, on avait admis qu'il serait possible de débarrasser suffisamment le bief de Chèvres des dépôts d'alluvions en adoptant un régime de "retenue réduite " consistant à abaisser le niveau de la retenue en fonction du débit, la pente du lit du Rhône le permettant à la faveur des crues. Rapidement, ce procédé se trouva en échec. Comme l'a fait remarquer très justement $\mathrm{M}$. SAUGEY, directeur de l'usine de Chèvres, la fréquence des crues extraordinaires est beaucoup trop faible pour assurer le curage du lit.

Il était donc indispensable de recourir à des chasses, c'est-à-dire l'abaissement total de la retenue et l'ouverture complète des vannes du barrage permettant l'établissement d'un débit de chasse dans le chenal naturel du Rhône.

(1) Aménagement de la chute du Rhône entre le confluent de l'Arve de Russin (7 nov. 1929). Rapport de M. I. Willhelm, ingénieur en chef des ponts et chaussées.

\section{The solids alluvial deposits from the river Arve into the Geneva's Rhône}

The dammings on the Rhône, downstream the lake of Geneva, are in serious danger of obturation, owing to solids alluvial deposits from the river Arve. Flushings regulary practiced, have enabled to maintain the Rhône channel close to its natural state. A wide exploitation retrospective over several tens of years is represented for the dammings of: Chèvres (1896-1942), Verbois (1942-1987), Chancy-Pougny (19241987). 
tableAU No 1: VARIATION DES VOLUMES - RETENUE, ALLUVION DEPOSEE

\begin{tabular}{|c|c|c|c|c|c|c|}
\hline \multirow{2}{*}{$\begin{array}{c}\text { Chasse } \\
\text { No }\end{array}$} & \multirow{2}{*}{ Annee } & \multicolumn{2}{|c|}{ Volume de retenue en $10^{6} \mathrm{~m}^{3}$} & \multicolumn{2}{|c|}{$\begin{array}{c}\text { Volume alluvion en } \\
10^{3} \mathrm{~m} 3\end{array}$} & \multirow{2}{*}{$\begin{array}{c}\text { Volume } \\
\text { residue } \\
10^{3} \mathrm{~m}^{3}\end{array}$} \\
\hline & & avant chasse & après chasse & $\begin{array}{c}\text { depowin } \\
\text { chasse } \\
\text { procecsente }\end{array}$ & $\begin{array}{l}\text { tevacuet } \\
\text { parchasse }\end{array}$ & \\
\hline$\overline{0}$ & & 14.950 & & & & \\
\hline 1 & 1945 & 13.953 & 14.765 & 997 & 812 & 185 \\
\hline 2 & 1947 & 13.855 & 14.470 & 910 & 615 & 295 \\
\hline 3 & 1949 & 13.724 & 14.314 & 746 & 590 & 156 \\
\hline 4 & 1951 & 13.074 & 14.126 & 1240 & 1052 & 188 \\
\hline 5 & 1954 & 13.182 & 13.847 & 944 & 665 & 279 \\
\hline 6 & 1956 & 13.036 & 13.834 & 811 & 798 & 13 \\
\hline 7 & 1960 & 11.989 & 13.175 & 1845 & 1186 & 659 \\
\hline 8 & 1965 & 10.426 & 12.566 & 2749 & 2140 & 609 \\
\hline 9 & 1969 & 11.268 & 12.703 & 1298 & 1435 & -137 \\
\hline 10 & 1972 & 11.580 & 12.979 & 1123 & 1399 & -276 \\
\hline 11 & 1975 & 11.600 & 13.39811 & 1379 & 1798 & -419 \\
\hline 12 & 1978 & 12.305 & 13.327 & 1093 & 1022 & 71 \\
\hline 13 & 1981 & 11.291 & 12.033 & 2036 & 742 & 1294 \\
\hline 14 & 1984 & 11.514 & 12.21821 & $5193)$ & 87931 & $.3603)$ \\
\hline
\end{tabular}

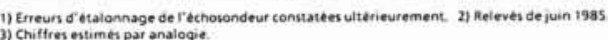

TABLEAU NO 2 ENVASEMENT ET CHASSES - INDICES CARACTERISTIQUES

\begin{tabular}{|c|c|c|c|c|c|c|c|}
\hline \multirow[b]{2}{*}{$\begin{array}{c}\text { Chasse } \\
\text { No }\end{array}$} & \multirow[b]{2}{*}{ Annee } & \multicolumn{3}{|c|}{ Envasement } & \multirow{2}{*}{$\begin{array}{l}\begin{array}{c}\text { Rendement } \\
\text { chase }\end{array} \\
\%\end{array}$} & \multirow{2}{*}{$\begin{array}{l}\text { Dilution } \\
\text { o/oo }\end{array}$} & \multirow{2}{*}{$\begin{array}{c}\text { Débit } \\
\text { Arve } \\
\text { Debit } \\
\text { chasse } \\
\text { st }\end{array}$} \\
\hline & & $\begin{array}{l}\text { EMA } 13 \\
103 \mathrm{mi} / \mathrm{an}\end{array}$ & $\begin{array}{c}\text { avant } \\
\text { chasse } 21 \\
*\end{array}$ & $\begin{array}{c}\text { apres } \\
\text { chasse 21 } \\
36\end{array}$ & & & \\
\hline 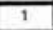 & 1945 & 332 & 6.65 & 1.22 & $\overline{82}$ & 14.7 & 21 \\
\hline 2 & 1947 & 405 & 7.31 & 3.20 & 68 & 14.2 & 17 \\
\hline 3 & 1949 & 373 & 8.20 & 4.24 & 79 & 16.4 & 77 \\
\hline 4 & 1951 & 620 & 12.53 & 5.50 & 85 & 19.4 & 48 \\
\hline 5 & 1954 & 315 & 1181 & 7.36 & 70 & 17.5 & 23 \\
\hline 6 & 1956 & 406 & 12.80 & 7.45 & 98 & 24.7 & 14 \\
\hline 7 & 1960 & 461 & 19.80 & 11.86 & 64 & 24.4 & 25 \\
\hline 8 & 1965 & 550 & 3025 & 15.93 & 78 & 366 & 28 \\
\hline 9 & 1969 & 325 & 24.62 & 15.01 & 111 & 15 & 18 \\
\hline 10 & 1972 & 374 & 22.53 & 13.17 & 125 & 20 & 24 \\
\hline 11 & 1975 & 460 & 22.40 & 10.36 & 130 & 25.8 & 26 \\
\hline 12 & 1978 & 364 & 17.68 & 10.84 & 93 & 15.3 & 44 \\
\hline $\left.13^{3}\right)$ & 1981 & 400 & 19.53 & 14.56 & 62 & 12.8 & 14 \\
\hline 143$)$ & 1984 & 467 & 23.90 & 16.57 & 79 & 104 & 27 \\
\hline
\end{tabular}

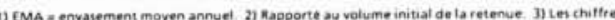
pour ces chasses ont ete corrigees - vair tente pour esplications
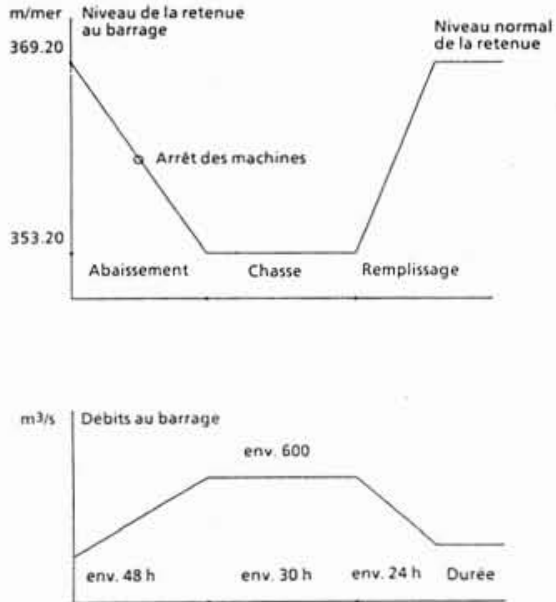

1. Diagramme des niveaux et des débits caractéristiques de chasse.

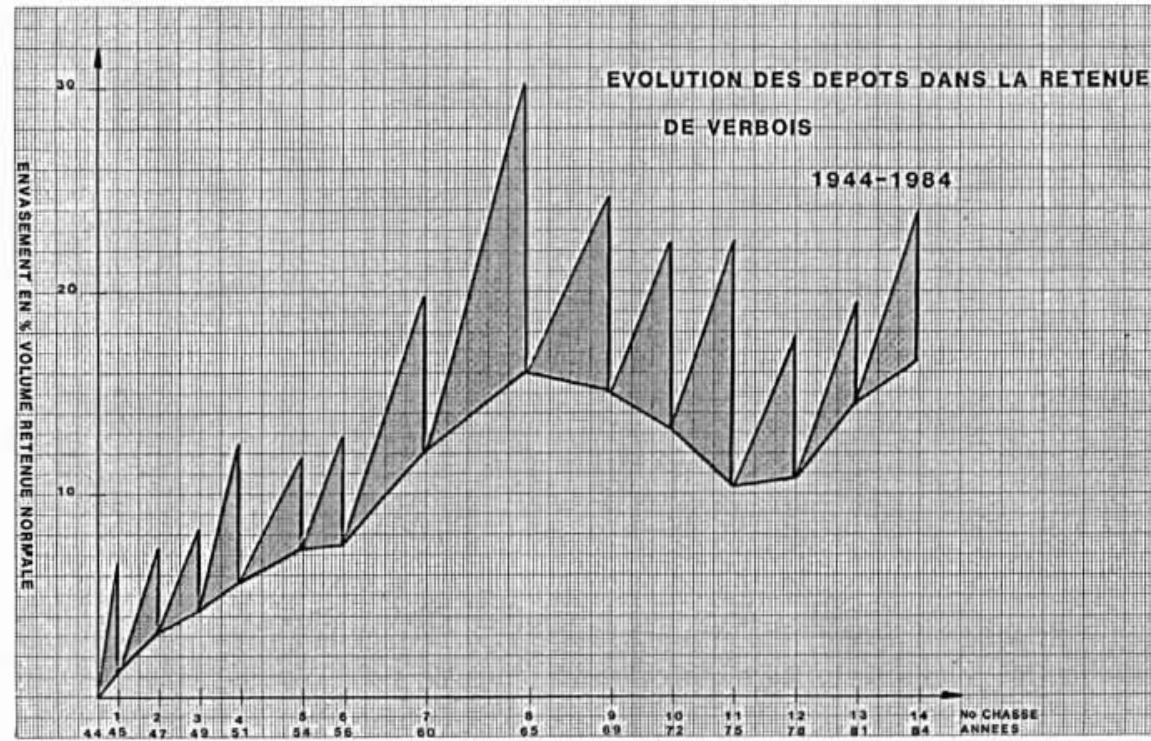

2.

\subsection{Les chasses de Verbois}

Dès 1913, la pratique des chasses fut systématiquement introduite à Chèvres ; ce bief de $7000 \mathrm{~m}$ de long a fait office de banc d'essai pendant 30 ans en matière de lutte contre l'alluvionnement.

Dans les années 1930, des études très poussées ont été faites sur l'alluvionnement en prévision de la construction de l'usine III qui s'appellera Verbois. De grandes personnalités, telles que les professeurs LUGEON, JouKOWSKY, MEYer-Peter, se sont attachées aux problèmes du transport des alluvions et de l'envasement de la retenue de Verbois. Les indications de M. le professeur MEYER-PETER (1930) concernant l'organisation des chasses et l'utilisation du Lac Léman comme réservoir sont simples et ingénieuses. L'énergie mécanique de l'eau serait suffisante pour affouiller et entrainer tous les alluvions déposés.

\section{Mécanisme (figure 1)}

1) La première phase consiste en l'abaissement très progressif de la cote de la retenue jusqu'à atteindre le niveau naturel du Rhône, durée environ 48 h (débit environ $300 \mathrm{~m}^{3} / \mathrm{s}$ ).

2) La purge ou chasse est alors enclenchée par lâchures issues du Léman jusqu'à concurrence de $600 \mathrm{~m}^{3} / \mathrm{s}$, durée $30 \mathrm{~h}$.

3) Remontée de la retenue dans un délai de $24 \mathrm{~h}$ environ. 


\subsection{Les matériaux charriés}

Les apports de l'Arve étaient estimés par le professeur COllet au début du siècle dans les limites suivantes: matériaux en suspension entre 1 à 2,4 millions de $\mathrm{m}^{3}$ par année.

Les graviers ont fait l'objet d'études particulières par MM. Lugeon, Collet, Saugey. On admettait que le volume annuel maximum des graviers charriés par l'Arve était de $200000 \mathrm{~m}^{3}$, le chiffre moyen annuel s'élevait à 160000 .

Côté Léman, aucun indice de charge de l'eau n'est signalé à une époque ou à une autre. Les apports sont assimilés à de l'eau claire ce qui est tout relatif.

\subsection{Les rejets des chasses (tableau $n^{\circ} 1$ )}

L'évaluation de la quantité de matériaux transitée par la chasse se fait par la méthode des relevés de profils sur le bief amont effectués avant et après la chasse. Les chiffres ainsi calculés varient beaucoup d'une chasse à l'autre, le plus petit est de $615000 \mathrm{~m}^{3}$ en 1947 ; le plus grand de $2140000 \mathrm{~m}^{3}$ lors de la chasse de 1965 espacée de la précédente de 5 ans, il faut le souligner.

Les facteurs principaux agissant sur l'importance de ces rejets sont: la durée de la chasse, le débit et l'intervalle entre chasses.

Des phénomènes transitoires (manœuvres) provoquant des affouillements localisés et intenses peuvent modifier le caractère linéaire de ces facteurs, de même que le durcissement (pavage) localisé de certains dépôts.

\subsection{Envasement}

Sur la base des relevés effectués pour chaque chasse de la retenue de Verbois, on note un envasement moyen annuel variant entre un minimum de $315000 \mathrm{~m}^{3}$ et $620000 \mathrm{~m}^{3}$ au maximum. Le volume de la retenue de Verbois initialement de 15 millions de $\mathrm{m}^{3}$ a régulièrement diminué du fait des dépôts qui se sont formés latéralement ; ceux-ci représentent actuellement $15 \%$ du volume de la retenue. La figure 2 montre l'évolution de ces dépôts.

\subsection{Fréquence des chasses}

Pendant la période d'exploitation de l'usine de Chèvres qui va de 1895 à 1942 (47 ans) les chasses ont été fréquemment pratiquées selon deux procédures distinctes; par abaissement partiel dans un premier temps puis en évacuation totale de la retenue par la suite, celle-ci étant reconnue comme la plus efficace.

Entre 1899 et 1929, M. Saugey, chef de l'usine de Chèvres, a indiqué que 17 campagnes de chasse ont été menées; ce qui donne un espacement moyen de moins de 2 ans entre chaque chasse.

Les exploitants de Verbois ont repris ce même rythme au début puis divers essais ont été pratiqués comme on peut le voir dans le tableau récapitulatif $\mathrm{n}^{\circ} 1$, on a même attendu 5 ans après la chasse de 1960 . Sur le plan de la pollution, on a qualifié cette chasse (1965) de désastreuse.

Depuis 1969, un intervalle de 3 ans a été régulièrement observé suite à un accord franco-suisse établi le 4 octobre 1967.

\subsection{Efficacité des chasses}

Le but étant bien compris, l'efficacité devient la préoccupation des exploitants. Il faut assurer le transit des apports solides de l'Arve, sinon un seuil se constitue à la Jonction et favorise des débordements à la première crue.

En quoi une chasse est-elle plus efficace qu'une autre? Par la quantité de matériaux qu'on a pu évacuer. Comme on l'a vu précédemment, les chiffres sont très variables, les causes agissantes étant nombreuses ; on note principalement :

- les apports variables de l'Arve pendant les trois années qui précèdent la chasse ;

- le débit de chasse que nous essayons de maintenir constant à $600 \mathrm{~m}^{3}$ mais qui est régulièrement perturbé par les variations de l'Arve en cette saison toujours fertile en crues. Une crue n'est pas forcément un bienfait malgré l'apport quantitatif en eau qu'elle présente; elle entraîne aussi une augmentation de la turbidité de l'eau; elle contraint les exploitants à des manœuvres fréquentes mais fort délicates au niveau des vannes de barrages dans le but de respecter le programme horaire très strict convenu à l'avance entre les responsables d'usine. Une crue de l'Arve survenant durant les $48 \mathrm{~h}$ d'abaissement de la retenue de Verbois provoque plus de mal que de bien, elle produit une attaque inopportune des talus de limon à des niveaux qui ne présentent aucun intérêt, il s'ensuit des effondrements et même des dégâts aux ouvrages.

Dans le tableau $n^{\circ} 2$, on a représenté sur la $6^{\mathrm{e}}$ colonne des chiffres qui reflètent cette efficacité (improprement qualifié de rendement) c'est le volume de matériaux évacué par la chasse, rapporté au volume déposé depuis la précédente, en pour cent.

\section{Atteintes aux berges}

Les berges sont des éléments frontières entre les deux milieux: mobile l'eau, immobile le terrain. Assurément, elles sont sollicitées en phase de vidange par le phénomène de décompression des nappes superficielles alimentées par la retenue. Dans les zones naturellement instables de Peney par exemple, la présence de la retenue constitue un frein à l'écroulement ; durant la vidange les mouvements reprennent très sensiblement.

Les talus de limon qui se sont déposés sont aussi des éléments instables. Ils ont été formés progressivement dans les zones à faible courant depuis 1942. A certains endroits, ils affleurent et des roselières ont pris naissance, elles sont très fréquentées par la faune. Nous cherchons à éviter leur désagrégation et pratiquons toutes menœuvres de variation de débit et de niveau de façon très lente et progressive. 
Les ouvrages évacuateurs d'eau et les structures de soutènement qui sont normalement immergés par la retenue sont aussi fort sensibles aux variations de niveau et de débit. Tous nos efforts tendent à les préserver en pratiquant des manœuvres lentes et contrôlées.

\section{Atteintes à la biocénose}

Elles sont diversement appréciées et interprétées :

Le phénomène de chasse sur la faune est indéniable ; bon nombre d'espèces se réfugient dans les cours d'eau affluents, d'autres résistent aux vitesses d'eau qui ne sont pas très différentes de celles qu'on voit en phase de crue dans l'Arve par exemple.
La turbidité de l'eau provoque certainement des dégâts dans le système respiratoire de nombreuses espèces. Il faut noter que certaines crues de l'Arve atteignent des taux de charge des eaux assez semblables.

Pour ce qui est des autres facteurs qui caractérisent la santé de l'eau et des aliments destinés à la faune, de nombreux développements ont été présentés dans le contexte des vidanges et des chasses par les spécialistes en la matière.

Pour certains composés organiques, la chasse constitue une action dépurative qui ne peut être néfaste.

Max Cohen,
Ingénieur,
Genève

\section{Conditions hydrauliques à l'aval du barrage de Chancy-Pougny}

\subsection{Etat actuel}

A l'aval de l'usine de Chancy-Pougny, le Rhône coule encore dans son état naturel et n'est pas influencé par la retenue de l'aménagement situé plus en aval. En effet, l'origine du remous de la retenue de Génissiat ne s'étend pas au-delà du point frontière, au lieu-dit « L'Etournel ".

Ce secteur du Rhône, d'une longueur d'environ $3,85 \mathrm{~km}$, compris entre ce point et l'usine de Chancy-Pougny présente encore tous les aspects d'un fleuve non aménagé avec une pente de l'ordre de $0,17 \%$ et une largeur moyenne d'environ $80 \mathrm{~m}$. En conséquence, les vitesses et la capacité de charriage sont assez élevées.

Une importante érosion du lit s'est produite au fil des années. Il s'est approfondi en moyenne d'environ $1,50 \mathrm{~m}$. Deux phénomènes combinés sont à la source de ces érosions : l'absence de matériaux naturels charriés depuis l'amont, ainsi que les dragages importants effectués à la naissance de la retenue de Génissiat, dans la région de l'Etournel. Dans les zones draguées, la pente du lit du fleuve augmente localement, alors que l'absence de matériaux solides de charriage ne permet pas de combler ces zones d'emprunts. Il s'ensuit une progression du phénomène d'érosion vers l'amont. Notons en outre que le manque de charriage tend à diminuer naturellement la pente du cours d'eau.

Bien que tous les 3 ans la purge de Verbois soit effectuée dans le but de chasser les envasements vers l'aval, cette mesure n'est pas suffisante pour arrêter l'évolution du phénomène d'érosion. En effet, les levés du fond du lit effectués à l'aval immédiat du barrage de Chancy-Pougny montrent que, si les affouillements au pied de l'ouvrage se remplissent bien de matériaux lors des purges, ces derniers sont ensuite à nouveau emportés en aval lors des décharges courantes du barrage.

L'érosion prend naissance à l'origine du remous de Génissiat pour graduellement s'accentuer en remontant vers l'amont. Au pied du barrage de Chancy-Pougny, le niveau du plan d'eau s'est abaissé d'environ $1,75 \mathrm{~m}$ entre 1924 et 1981 (voir courbes 8 et 9 de la figure 3). Par ailleurs, elle est toujours en progression comme en témoignent les courbes (10a) et (10b) de la même figure.

Les niveaux d'eau à l'aval du barrage, représentés par la courbe (9) de la figure 3 n'ont été mesurés que pour des débits allant jusqu'à $1000 \mathrm{~m}^{3} / \mathrm{s}$. Afin de déterminer d'une manière fiable les niveaux d'eau pour des débits supérieurs, un calcul de courbe de remous a été effectué partant de la relation débit - niveau d'eau à la station hydrométrique fédérale aux Rippes (courbe (10a)). L'avantage de ce calcul consiste dans le fait que les éventuelles erreurs commises lors de l'extrapolation des niveaux aux Rippes ne se répercutent que d'une manière atténuée aux niveaux pris plus en amont.

La conception originale du radier du barrage est également une cause d'affouillements importants au pied du barrage. Du fait qu'il n'y a, pratiquement pas de bassin amortisseur, la dissipation d'énergie se fait dans un bassin naturel formé sous l'action des eaux. Comme il fallait s'y attendre, l'affouillement a atteint la roche sous-jacente, c'est-à-dire la molasse dont le toit se trouve au niveau 325 environ. Les marnes couvrant la molasse, décrites dans les rapports géologiques comme étant de faible résistance, se dilatant et s'effritant aisément en surface, ne pouvaient résister, à la longue, aux sollicitations.

Les affouillements relevés sont représentés dans la figure 4 . Une coupe dans la passe $n^{\circ} 4$, une autre dans la tête d'écluse, ainsi que des profils en travers situés à $3 \mathrm{~m}$ et 


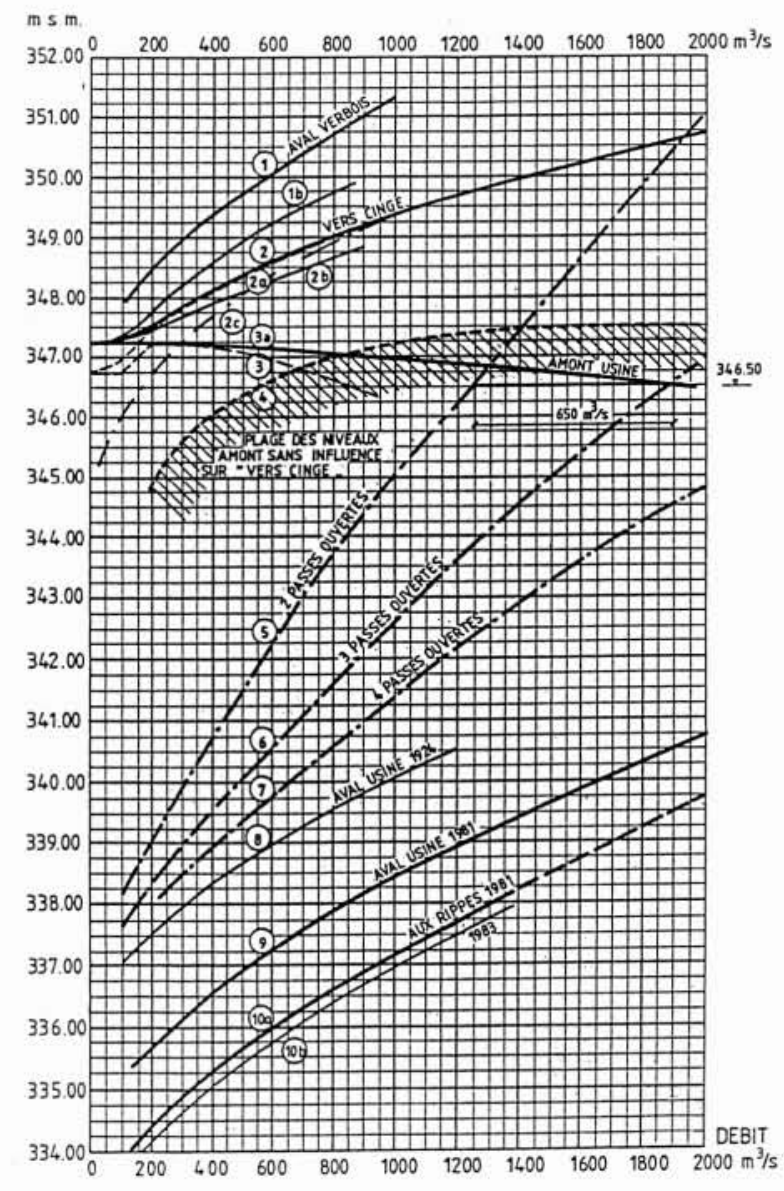

3. Niveaux d'eau en fonction des débits du Rhône aux endroits caractéristiques.

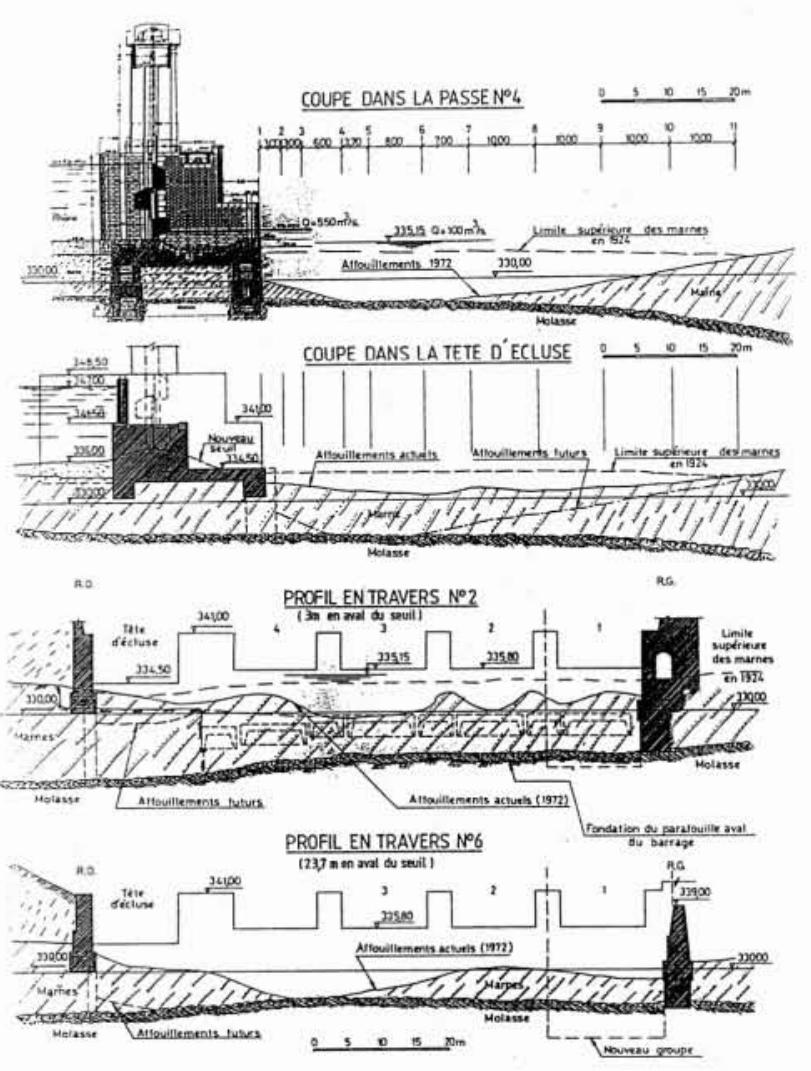

4. Affouillements actuels et futurs au pied du barrage.
$23,7 \mathrm{~m}$ en aval du seuil du barrage, donnent bien les dimensions de ces bassins naturels. Pour la représentation de cette situation, on a choisi les levés effectués en 1972 qui donnaient les profondeurs les plus caractéristiques et aussi les plus importantes.

Afin de caractériser l'écoulement en aval du barrage, relevons que, pour des débits importants, la vitesse au seuil atteint $12,5 \mathrm{~m} / \mathrm{s}$. Elle se réduit à $5 \mathrm{~m} / \mathrm{s}$ environ au droit de l'affouillement maximum. Dans le profil situé au bout de l'éperon mitoyen entre centrale et barrage, la vitesse d'écoulement s'élève encore approximativement à $3,5 \mathrm{~m} / \mathrm{s}$.

La zone d'eau morte à l'aval de la tête d'écluse, obturée dès sa construction dans l'attente de la réalisation d'une éventuelle écluse, a tendance à provoquer une déviation du courant vers la rive droite. Un courant de retour peut se former dans certains cas selon la disposition des passes ouvertes. Des vitesses de sens inverse peuvent alors être observées le long du mur rive droite. C'est ce courant de retour qui est responsable des affouillements que l'on constate déjà actuellement à l'aval de la tête d'écluse (fig. 3 et 4).

La berge sur la rive droite a été renforcée en 1981 au moyen d'enrochements. Ces blocs, dont les $2 / 3$ possèdent un poids supérieur à $1500 \mathrm{~kg}$ (ce qui correspond à un diamètre d'environ $1 \mathrm{~m}$ ), peuvent résister à des vitesses de l'ordre de $6,5 \mathrm{~m} / \mathrm{s}$.

\subsection{Ecrans mobiles dans l'aspirateur des groupes généra- teurs}

En 1948, constatant l'approfondissement sensible du lit du Rhône en aval de la centrale, les aspirateurs des groupes $\mathrm{n}^{\circ} 3,4$ et 5 ont été équipés d'écrans mobiles, ceci dans le but d'éviter toute entrée d'air pour les débits jusqu'à $200 \mathrm{~m}^{3} / \mathrm{s}$. Ces écrans, ayant pour mission de couper la lame d'eau à la sortie de l'aspirateur, évitent ainsi le désamorçage des turbines.

Depuis lors, cet approfondissement aval s'est poursuivi au cours des ans, tant et si bien qu'en 1980, il est nécessaire d'avoir recours à un équipement semblable à celui décrit ci-devant pour l'un des deux groupes restant pour l'exploitation de la centrale avec des débits jusqu'à $270 \mathrm{~m}^{3} / \mathrm{s}$, tout particulièrement lors de l'indisponibilité d'un groupe. Lors de cette deuxième intervention, en 1980, les quatre écrans mobiles ont été munis d'une commande électrique motorisée. Ces engins sont pilotés à partir de la salle de commande, en fonction manuelle. Ils évoluent entre la cote haute de : $335,80 \mathrm{msm}$ et la cote basse de : $334,60 \mathrm{msm}$. 